\title{
The Sigmoid Colon of Ulcerative Colitis
}

\author{
Akira Hokama · Yasushi Ihama · Hiroshi Chinen · \\ Kazuto Kishimoto $\cdot$ Fukunori Kinjo $\cdot$ Jiro Fujita
}

Published online: 3 March 2009

(C) Springer Science+Business Media, LLC 2009

A 70-year-old man with a 20-year history of ulcerative colitis was referred to our emergency unit for haematochezia of 2 weeks' duration. Physical examination showed localized tenderness in the left lower quadrant without rebound. Plain abdominal radiography showed the sigmoid colon with foreshortening and a loss of haustra (Fig. 1, arrowheads), indicating active ulcerative colitis. His condition got worse despite intensive medical treatments, and he thus underwent total colectomy and end ileostomy with uneventful recovery.

Although recent imaging studies have shown progress for assessing intestinal diseases, the plain abdominal radiograph is still crucial for evaluating the severity of ulcerative colitis, especially toxic megacolon, a wellknown life-threatening complication. Potential exacerbating factors of ulcerative colitis include colonoscopy and barium enema. In addition to toxic megacolon, we highlight that the tubular appearance on plain abdominal radiographs is quite helpful for assessing the activity of ulcerative colitis in emergency situations.

A. Hokama $(\bowtie)$. Y. Ihama · H. Chinen - K. Kishimoto ·

F. Kinjo $\cdot$ J. Fujita

First Department of Internal Medicine, University of the Ryukyus, 207 Uehara, Nishihara, Okinawa 903-0215, Japan e-mail: hokama-a@med.u-ryukyu.ac.jp

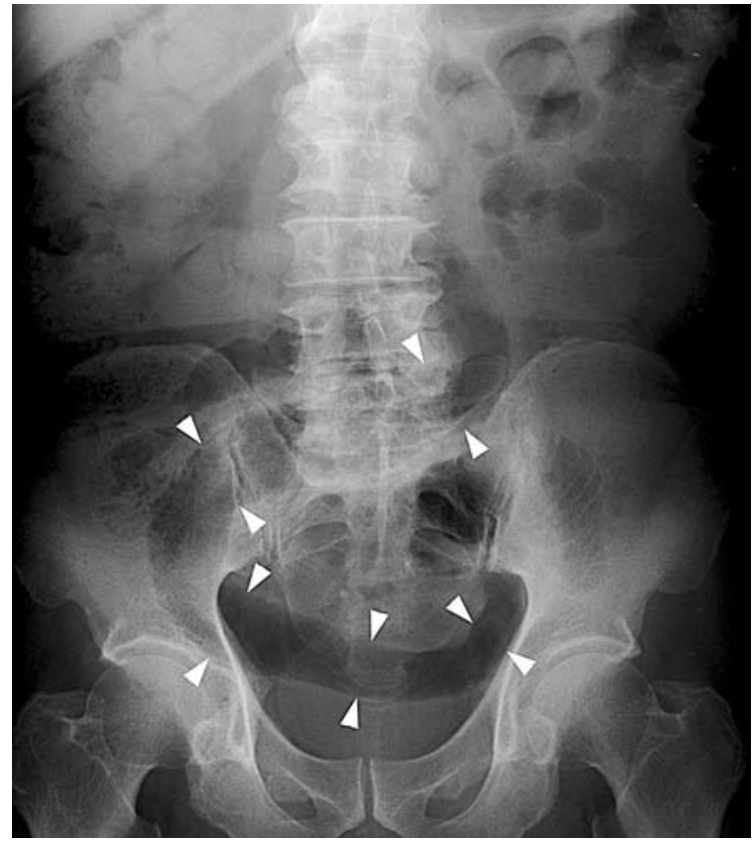

Fig. 1 Plain abdominal radiograph showing the sigmoid colon with foreshortening and a loss of haustra (arrowheads) 